\title{
Correction \\ Correction: Hu et al. Profiles of Long Non-Coding RNAs and mRNA Expression in Human Macrophages Regulated by Interleukin-27. Int. J. Mol. Sci. 2019, 20, 6207
}

\author{
Xiaojun $\mathrm{Hu}^{\dagger}$, Suranjana Goswami, Ju Qiu, Qian Chen, Sylvain Laverdure $\mathbb{D}^{\text {, }}$ Brad T. Sherman and \\ Tomozumi Imamichi *(D)
}

check for updates

Citation: Hu, X.; Goswami, S.; Qiu, J.; Chen, Q.; Laverdure, S.; Sherman, B.T.; Imamichi, T. Correction: Hu et al. Profiles of Long Non-Coding RNAs and mRNA Expression in Human Macrophages Regulated by Interleukin-27. Int. J. Mol. Sci. 2019, 20, 6207. Int. J. Mol. Sci. 2021, 22, 5129. https://doi.org/10.3390/ijms 22105129

Received: 15 April 2021 Accepted: 26 April 2021 Published: 12 May 2021

Publisher's Note: MDPI stays neutral with regard to jurisdictional claims in published maps and institutional affiliations.

Copyright: (c) 2021 by the authors. Licensee MDPI, Basel, Switzerland. This article is an open access article distributed under the terms and conditions of the Creative Commons Attribution (CC BY) license (https:/ / creativecommons.org/licenses/by/ $4.0 /)$.
Laboratory of Human Retrovirology and Immunoinformatics, Frederick National Laboratory for Cancer Research, Frederick, MD 21702, USA; xiaojun.hu@usda.gov (X.H.); suranjana.goswami@nih.gov (S.G.); ju.qiu@nih.gov (J.Q.); chenq3@mail.nih.gov (Q.C.); sylvain.laverdure@nih.gov (S.L.); bsherman@mail.nih.gov (B.T.S.)

* Correspondence: timamichi@mail.nih.gov; Tel.: +1-301-846-5450; Fax: +1-301-846-6762

+ Current affiliation: Plant Germplasm Quarantine Program, United States Department of Agriculture, Beltsville, MD 20705, USA.

The authors wish to make the following corrections to this paper [1]:

We have recently been made aware by Danielle and Jean Thierry-Mieg (NCBI. NLM. $\mathrm{NIH}$ ) of some errors and omissions in the Results section of our recent paper. The first paragraph of said Results currently reads as follows:

Finally, 2691 candidate lncRNA transcripts were obtained (Table S2). Among them, $53 \%$ were from intergenic regions, $27 \%$ from intronic regions, and $20 \%$ were antisense to protein-coding loci.

To set straight the scientific record, we would like to make the following corrections:

After removing ribosomal RNAs, 2613 candidate lncRNA transcripts were obtained (Table S2). Among them, 51\% were from intergenic regions, 28\% from intronic regions, and $21 \%$ were antisense to protein-coding loci.

The authors declare that this change does not affect the validity of the conclusion. The authors would like to apologize for any inconvenience caused to the readers by these changes.

Supplementary Materials: The following are available online at https://www.mdpi.com/article/10 .3390/ijms22105129/s1.

Funding: This project has been funded in whole or in part with federal funds from the National Cancer Institute, and the National Institute of Allergy and Infectious Disease, National Institutes of Health, under Contract No. HHSN261200800001E.

Institutional Review Board Statement: Approval for these studies, including all sample materials, was granted by the National Institute of Allergy and Infectious Diseases Institutional Review Board. All experimental procedures in these studies were approved by the National Cancer Institute at Frederick and Frederick National Laboratory for Cancer Research, and were performed in accordance with the relevant guidelines and regulations (the protocol code number: 16-19, approval data: 6 January 2017).

Data Availability Statement: The data presented in this study are openly available in the NCBI SRA accession no. PRJNA559359.

Conflicts of Interest: The authors declare no conflict of interest.

\section{Reference}

1. Hu, X.; Goswami, S.; Qiu, J.; Chen, Q.; Laverdure, S.; Sherman, B.T.; Imamichi, T. Profiles of Long Non-Coding RNAs and mRNA Expression in Human Macrophages Regulated by Interleukin-27. Int. J. Mol. Sci. 2019, 20, 6207. [CrossRef] 